\title{
Evaluation of WGS-subtyping methods for epidemiological surveillance of foodborne salmonellosis
}

Manal Mohammed ${ }^{*}$ id and Salina Thapa

\begin{abstract}
Background: Salmonellosis is one of the most common foodborne diseases worldwide. Although human infection by non-typhoidal Salmonella (NTS) enterica subspecies enterica is associated primarily with a self-limiting diarrhoeal illness, invasive bacterial infections (such as septicaemia, bacteraemia and meningitis) were also reported. Human outbreaks of NTS were reported in several countries all over the world including developing as well as high-income countries. Conventional laboratory methods such as pulsed field gel electrophoresis (PFGE) do not display adequate discrimination and have their limitations in epidemiological surveillance. It is therefore very crucial to use accurate, reliable and highly discriminative subtyping methods for epidemiological characterisation and outbreak investigation.
\end{abstract}

Methods: Here, we used different whole genome sequence (WGS)-based subtyping methods for retrospective investigation of two different outbreaks of Salmonella Typhimurium and Salmonella Dublin that occurred in 2013 in UK and Ireland respectively.

Results: Single nucleotide polymorphism (SNP)-based cluster analysis of Salmonella Typhimurium genomes revealed well supported clades, that were concordant with epidemiologically defined outbreak and confirmed the source of outbreak is due to consumption of contaminated mayonnaise. SNP-analyses of Salmonella Dublin genomes confirmed the outbreak however the source of infection could not be determined. The core genome multilocus sequence typing (cgMLST) was discriminatory and separated the outbreak strains of Salmonella Dublin from the non-outbreak strains that were concordant with the epidemiological data however CgMLST could neither discriminate between the outbreak and non-outbreak strains of Salmonella Typhimurium nor confirm that contaminated mayonnaise is the source of infection, On the other hand, other WGS-based subtyping methods including multilocus sequence typing (MLST), ribosomal MLST (rMLST), whole genome MLST (wgMLST), clustered regularly interspaced short palindromic repeats (CRISPRs), prophage sequence profiling, antibiotic resistance profile and plasmid typing methods were less discriminatory and could not confirm the source of the outbreak.

Conclusions: Foodborne salmonellosis is an important concern for public health therefore, it is crucial to use accurate, reliable and highly discriminative subtyping methods for epidemiological surveillance and outbreak investigation. In this study, we showed that SNP-based analyses do not only have the ability to confirm the occurrence of the outbreak but also to provide definitive evidence of the source of the outbreak in real-time.

Keywords: Salmonella, WGS, Subtyping, SNP-typing, Prophage profile, CRISPR typing, MLST, rMLST, wgMLST, cgMLST

\footnotetext{
* Correspondence: m.mohammed@westminster.ac.uk

School of Life Sciences, College of Liberal Arts and Sciences, University of Westminster, London, UK
}

(c) The Author(s). 2020 Open Access This article is licensed under a Creative Commons Attribution 4.0 International License, which permits use, sharing, adaptation, distribution and reproduction in any medium or format, as long as you give appropriate credit to the original author(s) and the source, provide a link to the Creative Commons licence, and indicate if changes were made. The images or other third party material in this article are included in the article's Creative Commons licence, unless indicated otherwise in a credit line to the material. If material is not included in the article's Creative Commons licence and your intended use is not permitted by statutory regulation or exceeds the permitted use, you will need to obtain permission directly from the copyright holder. To view a copy of this licence, visit http://creativecommons.org/licenses/by/4.0/. 


\section{Introduction}

Foodborne salmonellosis is an important concern for public health. It is caused by the enteric pathogen Salmonella enterica, which includes more than 2600 serovars [1]. Human Salmonella infections are classically divided into diseases caused by typhoidal or non-typhoidal salmonella (NTS). Typhoid fever is caused by the human restricted Salmonella enterica serovars Typhi and Paratyphi [2]. Although non-typhoidal Salmonella (NTS) serovars, predominantly cause a self-limiting diarrhoeal illness they have adapted to cause invasive extra-intestinal disease known as invasive NTS (iNTS) which can result in bacteraemia and focal systemic infections $[3,4]$. There are two licenced vaccines for prevention of typhoid fever however, they are not effective against NTS [5] moreover, management of iNTS illness is complicated by the emergence of multidrug resistant (MDR) strains [6]. Salmonella serovars responsible for typhoid fever kill over 250,000 humans per year [7] while non-typhoidal Salmonella (NTS) serovars responsible for diarrhoeal illness cause over 155,000 deaths annually [8]. Interestingly, NTS have adapted to cause febrile bacteraemia and serious systemic infections; it has been estimated that over 680,000 people die every year as a result of infection by invasive NTS (iNTS) [3]. Salmonella Typhimurium and Salmonella Dublin have been associated with systemic illness [4, 5]. Human outbreaks of Salmonella Typhimurium and Salmonella Dublin were reported in developed countries [9-11].

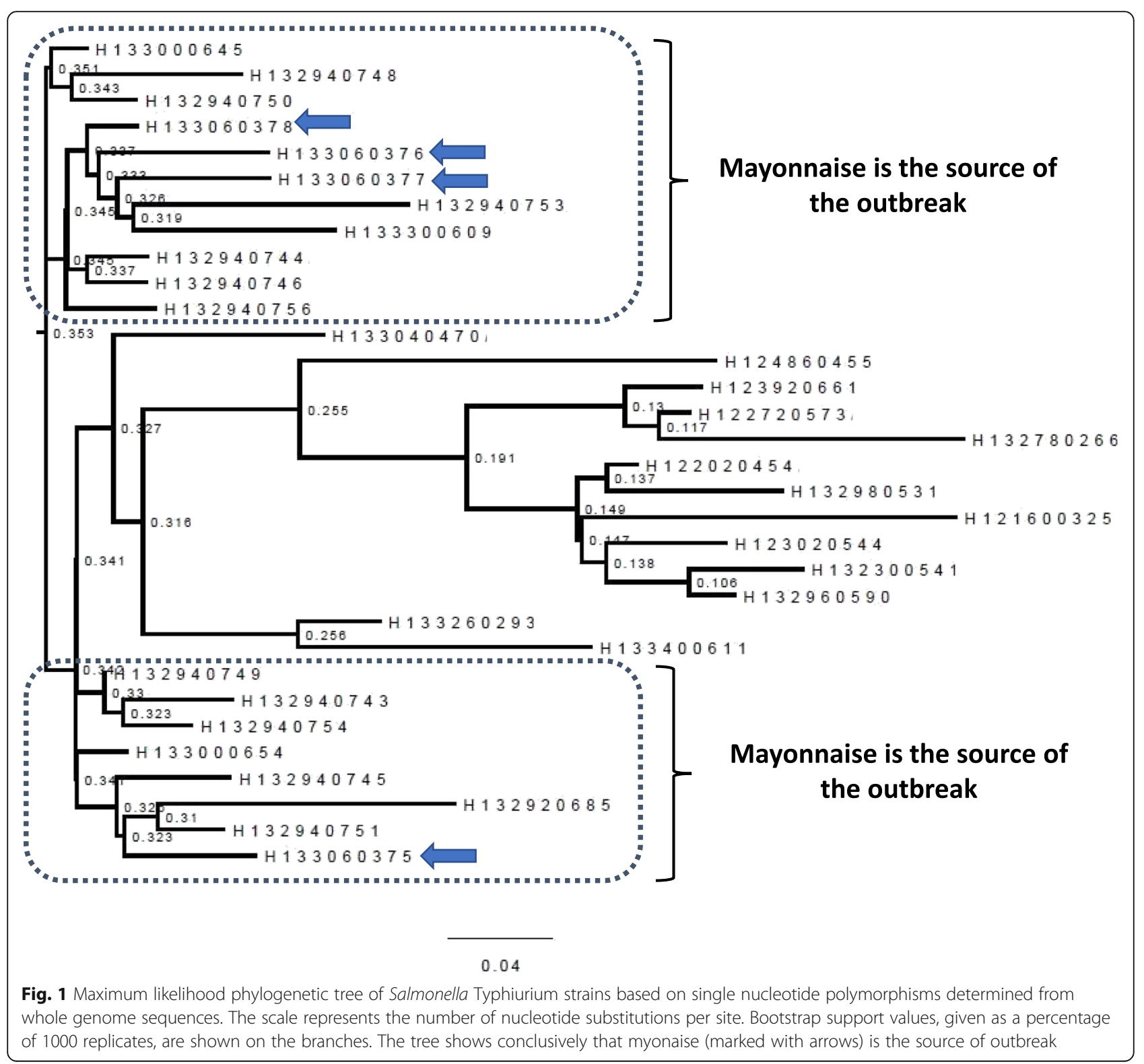


Conventional laboratory methods such as pulsed field gel electrophoresis (PFGE) do not usually provide adequate discrimination among outbreak and nonoutbreak strains of Salmonella enterica and have their limitations in epidemiological surveillance, it is therefore crucial to use accurate, reliable and highly discriminative subtyping methods for epidemiological characterisation and outbreak investigation.

Here, we evaluate different whole genome sequence (WGS)-based subtyping methods (including single nucleotide polymorphism (SNP)-based cluster analysis, multilocus sequence typing (MLST), ribosomal MLST (rMLST), whole genome MLST (wgMLST), core genome MLST (cgMLST) as well as clustered regularly interspaced short palindromic repeats (CRISPRs), prophage sequence profiling, antibiotic resistance profile and plasmid typing) for retrospective investigation of two outbreaks of Salmonella Typhimurium and Salmonella Dublin that occurred in 2013 in UK and Ireland respectively $[9,12]$.

\section{Methods}

Retrospective analyses of the two outbreaks of Salmonella Typhimurium and Salmonella Dublin

We carried out retrospective investigation of a human outbreak of Salmonella Dublin that occurred in 2013 in Ireland [9] and another human outbreak of Salmonella Typhimurium occurred in 2013 in UK [12]. We included suspected food strains isolated from mayonnaise and raw-milk cheeses that can be linked to the outbreaks of Salmonella Typhimurium and Salmonella Dublin respectively. Non-outbreak strains were also included for comparison. Details of all Salmonella Dublin and Salmonella Typhimurium isolates analysed in this study are provided in supplementary Tables 1 and 2 respectively.

PFGE was of a limited value for the investigation of the outbreak of Salmonella Dublin [9] since all outbreak and non-outbreak isolates of Salmonella Dublin were indistinguishable by PFGE. Although multiple loci VNTR analysis (MLVA) was of value in discriminating the

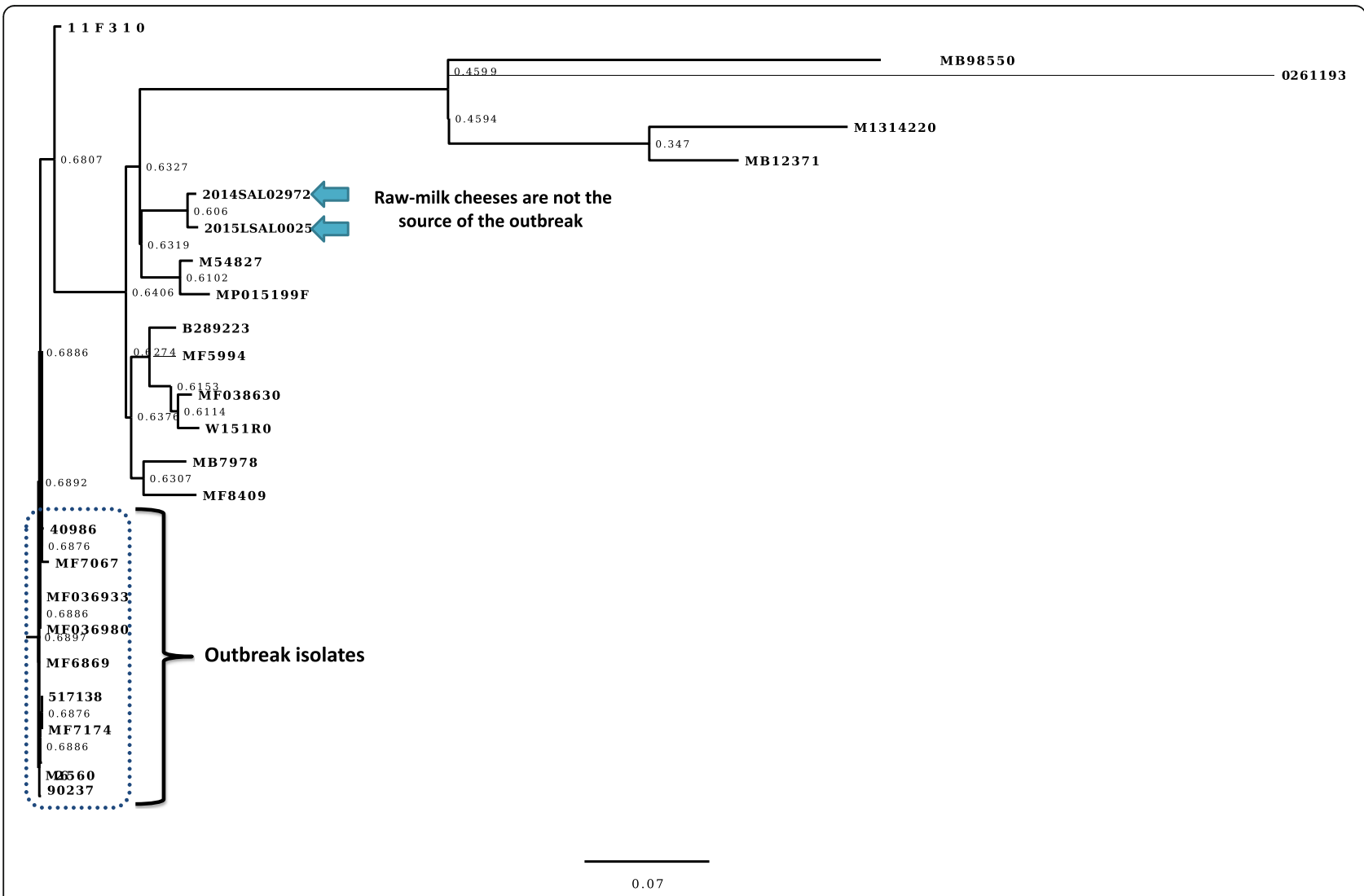

Fig. 2 Maximum likelihood phylogenetic tree of Salmonella Dublin strains based on single nucleotide polymorphisms determined from whole genome sequences. The scale represents the number of nucleotide substitutions per site. Bootstrap support values, given as a percentage of 1000 replicates, are shown on the branches. All Salmonella Dublin isolates had indistinguishable pulsed-field gel electrophoresis profiles. Confirmed outbreak cases $(n=9)$ in October-November 2013 are grouped together in one cluster. However, the source of the outbreak could not be determined as outbreak isolates showed high genetic divergence to bacterial strains isolated from the raw-milk cheeses (marked with arrows) including isolate 2014SAL02972 from Morbier cheese (accession number; ERS2767809) and isolate 2015LSAL00258 from St. Nectaire cheese (accession number: ERS2767808) 
outbreak strains from an epidemiologically unrelated isolate in 2013 it was not able to provide a conclusive link between the outbreak strain and a historical isolate from 2011 (11F310) since all outbreak strains had the same MLVA pattern (3-6-1-10-2-3-12) and the historical isolate had similar MLVA pattern (3-6-1-10-2-3-11/12).

Despite the technical limitation of phage typing, it was of value for investigating the outbreak of Salmonella Typhimurium [12] and confirming that mayonnaise is the source of infection.

Denovo assembly of WGS data of Salmonella Dublin and Salmonella Typhimurium strains

We carried out denovo assembly for the raw Fastq paired end (PE) reads for all Salmonella Dublin and Salmonella

Table 1 MLST, rMLST, cgMLST and wgMLST results of Salmonella Dublin outbreak and non-outbreak strains

\begin{tabular}{lllll}
\hline Strain ID: & MLST: & rMLST: & cgMLST: & wgMLST: \\
\hline $\begin{array}{l}\text { Outbreak strains: } \\
\text { 902,637 }\end{array}$ & 10 & 1429 & 38,665 & 259,116 \\
MF036933 & 10 & 1429 & 38,665 & 259,117 \\
MF036980 & 10 & 1429 & 38,665 & 259,118 \\
517,138 & 10 & 1429 & 38,665 & 259,121 \\
MF6869 & 10 & 1429 & 38,665 & 259,127 \\
M26560 & 10 & 1429 & 38,665 & 259,123 \\
MF7067 & 10 & 1429 & 38,665 & 259,122 \\
MF7174 & 10 & 1429 & 38,665 & 259,128 \\
40,986 & 10 & 1429 & 38,665 & 259,126
\end{tabular}

\section{Non-outbreak strains:}

$\begin{array}{lllll}\text { MF038630 } & 10 & 1429 & 38,666 & 259,131 \\ \text { M1314220 } & 10 & 26,829 & 38,664 & 259,120 \\ \text { M54827 } & 10 & 1429 & 38,667 & 259,129 \\ \text { MB12371 } & 10 & 26,829 & 38,668 & 259,130 \\ \text { MF5994 } & 10 & 92,451 & 38,669 & 259,145 \\ \text { MB7978 } & 10 & 1429 & 38,670 & 259,133 \\ \text { B289223 } & 10 & 1429 & 38,671 & 259,134 \\ \text { 11F310 } & 10 & 1429 & 38,655 & 259,135 \\ \text { MB98550 } & 10 & 3696 & 38,657 & 259,142 \\ \text { MF8409 } & 10 & 1429 & 38,658 & 259,139 \\ \text { W151R0 } & 10 & 1429 & 38,659 & 259,140 \\ \text { B261193 } & 10 & 92,450 & 38,660 & 259,141 \\ \text { MP015199F } & 10 & 1429 & 38,661 & 259,148 \\ \text { Food isolates: } & & & & \\ \text { a2014LSAL02972 } & 10 & 1429 & 230,922 & 283,421 \\ \text { a2015LSAL00258 } & 10 & 96,856 & 146,469 & 283,422\end{array}$

Same results for MLST, rMLST, cgMLST and wgMLST were obtained from CGE and Enterobase using Velvet and SPAdes assemblers respectively. ${ }^{a}$ Salmonella Dublin strains isolated from raw milk cheeses related to other outbreaks occurred in France [10]
Typhimurium strains using two different assemblers including Velvet available at Centre for genomic epidemiology (CGE) (http://www.genomicepidemiology.org/) and SPAdes available at Enterobase (http://enterobase. warwick.ac.uk/). We then assessed the quality of the

Table 2 MLST, rMLST, cgMLST and wgMLST results of Salmonella Typhimurium outbreak and non-outbreak strains

\begin{tabular}{|c|c|c|c|c|}
\hline Strain ID & MLST: & rMLST: & cgMLST: & wgMLST: \\
\hline \multicolumn{5}{|l|}{ Food strains: } \\
\hline${ }^{\mathrm{a}} \mathrm{H} 133060375$ & 19 & 1392 & 60,658 & 70,401 \\
\hline${ }^{\mathrm{a}} \mathrm{H} 133060376$ & 19 & 1392 & 60,660 & 70,402 \\
\hline 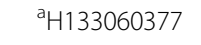 & 19 & 1392 & 36,749 & 70,514 \\
\hline${ }^{\mathrm{a}} \mathrm{H} 133060378$ & 19 & 1392 & 60,661 & 70,403 \\
\hline \multicolumn{5}{|c|}{ Outbreak strains: } \\
\hline H133000654 & 19 & 1392 & 36,749 & 70,398 \\
\hline H132940743 & 19 & 1392 & 36,749 & 70,404 \\
\hline H132940744 & 19 & 1392 & 60,662 & 70,405 \\
\hline H132940745 & 19 & 1392 & 60,663 & 70,406 \\
\hline H132940746 & 19 & 1392 & 36,749 & 70,431 \\
\hline H132940748 & 19 & 1392 & 60,683 & 70,432 \\
\hline H132940749 & 19 & 1392 & 36,749 & 70,433 \\
\hline H132940750 & 19 & 1392 & 60,684 & 70,439 \\
\hline H132940751 & 19 & 1392 & 60,685 & 70,440 \\
\hline H132940753 & 19 & 1392 & 61,002 & 70,834 \\
\hline H132940754 & 19 & 1392 & 36,754 & 70,835 \\
\hline H132940756 & 2392 & 1392 & 61,001 & 70,833 \\
\hline H133000645 & 19 & 1392 & 36,749 & \\
\hline H133300609 & 19 & 1392 & 36,749 & 70,944 \\
\hline H132300541 & 19 & 1391 & 36,751 & 70,951 \\
\hline \multicolumn{5}{|c|}{ Non-outbreak strains: } \\
\hline H133260293 & 19 & 1392 & 71,438 & 84,026 \\
\hline H132780266 & 19 & 1391 & 71,450 & 84,040 \\
\hline H132960590 & 19 & 1391 & 36,751 & 84,041 \\
\hline H132920685 & 19 & 1392 & 36,763 & 84,076 \\
\hline H132980531 & 19 & 1391 & 36,774 & 87,971 \\
\hline H121600325 & 19 & 1391 & 20,224 & 87,972 \\
\hline $\mathrm{H} 122720573$ & 19 & 1391 & 20,848 & 87,973 \\
\hline H12320661 & 19 & 1391 & 20,882 & 87,974 \\
\hline H123020544 & 19 & 1391 & 20,711 & 87,975 \\
\hline H122020454 & 19 & 1391 & 21,310 & 88,017 \\
\hline $\mathrm{H} 124860455$ & 19 & 26,127 & 20,800 & 88,018 \\
\hline $\mathrm{H} 133040470$ & 19 & 1392 & 71,422 & 84,006 \\
\hline H1330400611 & 19 & 1392 & 71,438 & 84,025 \\
\hline
\end{tabular}

${ }^{a}$ Strains of Salmonella Typhimurium isolated from mayonnaise Same results for MLST, rMLST, cgMLST and wgMLST were obtained from CGE and Enterobase using Velvet and SPAdes assemblers respectively. 
assembly for each strain was assessed using Quast assessment tool (http://quast.bioinf.spbau.ru/).

\section{SNP typing analyses of Salmonella Dublin and Salmonella Typhimurium outbreaks}

SNP analysis was carried out using CSIPhylogeny (https://cge.cbs.dtu.dk/services/CSIPhylogeny/) where raw reads were mapped to reference sequences (strain LT2 of Salmonella Typhimurium; accession number: AE006468 and strain CT_02021853 of Salmonella Dublin; accession number: CP001144) using BWA software (http://bio-bwa.sourceforge.net). The depth at each mapped position was calculated using genomeCoverageBed, which is part of BEDTools (https://bedtools.readthedocs.io/en/latest/). High quality SNPs were called using mpileup which is part of SAMTools (http://samtools.sourceforge.net). Genome mappings were then compared and an alignment of the SNPs are then created by concatenating the SNPs.

Table 3 Number of spacers within CRISPR1 locus in all Salmonella Dublin strains analysed in this study

\begin{tabular}{|c|c|c|}
\hline Strain ID: & $\begin{array}{l}\text { Spacers No. } \\
\text { (Velvet) }\end{array}$ & $\begin{array}{l}\text { Spacers No. } \\
\text { (SPAdes) }\end{array}$ \\
\hline \multicolumn{3}{|c|}{ Outbreak strains: } \\
\hline 902,637 & 5 & 5 \\
\hline MF036933 & 5 & 5 \\
\hline MF036980 & 5 & 5 \\
\hline 517,138 & 4 & 5 \\
\hline MF6869 & 5 & 5 \\
\hline M26560 & 5 & 5 \\
\hline MF7067 & 4 & 5 \\
\hline MF7174 & 5 & 5 \\
\hline 40,986 & 5 & 5 \\
\hline \multicolumn{3}{|c|}{ Non-outbreak strains: } \\
\hline MF038630 & 5 & 5 \\
\hline M1314220 & 5 & 5 \\
\hline M54827 & 3 & 3 \\
\hline MB12371 & 5 & 5 \\
\hline MF5994 & 5 & 5 \\
\hline MB7978 & 5 & 5 \\
\hline B289223 & 5 & 5 \\
\hline $11 F 310$ & 5 & 5 \\
\hline MB98550 & 4 & 4 \\
\hline MF8409 & 5 & 5 \\
\hline W151R0 & 4 & 5 \\
\hline B261193 & 3 & 3 \\
\hline MP015199F & 3 & 3 \\
\hline
\end{tabular}

A maximum likelihood (ML) phylogenetic tree was then created based on the concatenated alignment of the high quality SNPs.

\section{Determination of MLST, rMLST, cgMLST and wgMLST of Salmonella Dublin and Salmonella Typhimurium strains} The assembled sequences of each strain were analyzed to detect the MLST, rMLST, cgMLST and wgMLST

Table 4 Number of spacers within CRISPRs loci in all Salmonella Typhimurium strains analysed in this study

\begin{tabular}{ll}
\hline Strain ID & $\begin{array}{l}\text { Spacers No. } \\
\text { (Velvet \& SPAdes) }\end{array}$ \\
\hline
\end{tabular}

\section{Food strains:}

${ }^{\mathrm{a}} \mathrm{H} 133060375$

$\begin{array}{lll}9 & 13 & 9 \\ 9 & 13 & 9 \\ 9 & 13 & 9 \\ 9 & 13 & 9\end{array}$

${ }^{\mathrm{a}} \mathrm{H} 133060378$

Outbreak strains:

$\begin{array}{llll}\text { H133300609 } & 9 & 13 & 9 \\ \mathrm{H} 132940743 & 9 & 13 & 9 \\ \mathrm{H} 132940744 & 9 & 13 & 9 \\ \mathrm{H} 132940745 & 9 & 13 & 9 \\ \mathrm{H} 132940746 & 9 & 13 & 9 \\ \mathrm{H} 132940748 & 9 & 13 & 9 \\ \mathrm{H} 132940749 & 9 & 13 & 9 \\ \mathrm{H} 132940750 & 9 & 13 & 9 \\ \mathrm{H} 132940751 & 9 & 13 & 9 \\ \mathrm{H} 132940753 & 13 & 9 & 9 \\ \mathrm{H} 132940754 & 9 & 13 & 9 \\ \mathrm{H} 132940756 & 9 & 13 & 9 \\ \mathrm{H} 133000645 & 9 & 13 & 9 \\ \mathrm{H} 133000654 & 9 & 13 & \end{array}$

\section{Non-outbreak strains}

$\begin{array}{llll}H 121600325 & 9 & 13 & 9 \\ H 122020454 & 9 & 13 & 9 \\ H 122720573 & 9 & 13 & 9 \\ H 123020544 & 9 & 13 & 9 \\ H 123920661 & 9 & 13 & 9 \\ H 124860455 & 9 & 13 & 9 \\ H 132780266 & 9 & 13 & 9 \\ H 132920685 & 9 & 13 & 9 \\ H 132960590 & 9 & 13 & 9 \\ H 132980531 & 9 & 13 & 9 \\ H 133040470 & 9 & 13 & 9 \\ H 133260293 & 9 & 13 & 9 \\ H 133400611 & 9 & 13 & 9\end{array}$

${ }^{a}$ Strains of Salmonella Typhimurium isolated from mayonnaise 
available at Enetrobase (http://enterobase.warwick.ac.uk/ ) and CGE (http://www.genomicepidemiology.org/).

\section{Determination of prophage sequence profiles in}

Salmonella Dublin and Salmonella Typhimurium genomes Prophages were determined with the draft genomes generated by Velevt and SPAdes for all Salmonella Dublin and Salmonella Typhimurium strains using PHASTER (http://phaster.ca/).

We then used CSI phylogeny available at CGE (http://www.genomicepidemiology.org/) to construct a phylogenetic tree based on the SNPs of detected prophages. Phylogenetic trees were constructed using assembled genomes generated by Velvet and SPAdes assemblers to check if the assembly could affect the tree.

\section{Determination of CRISPRs within Salmonella Dublin and} Salmonella Typhimurium strains

Spacers sequence within the draft genomes of all Salmonella Dublin and Salmonella Typhimurium strains were characterized using CRISPRFinder (http://crispr.i2 bc.paris-saclay.fr/Server/).
Determination of plasmids within Salmonella Dublin and Salmonella Typhimurium strains

We determined the plasmids within the draft genomes of all Salmonella Dublin and Salmonella Typhimurium strains using the plasmid database; PLSDB (https://ccbmicrobe.cs.uni-saarland.de/plsdb/).

In silico analyses of antibiotic resistance within Salmonella Dublin and Salmonella Typhimurium strains

We determined acquired antibiotic resistance genes and mutations within the draft genomes of all Salmonella Dublin and Salmonella Typhimurium strains using ResFinder (https://cge.cbs.dtu.dk/services/ ResFinder/)

\section{Results}

WGS-based subtyping

SNP based cluster analyses

SNP based tree showed conclusively that the outbreak strains of Salmonella Typhimurium were grouped together in two clades and they are very closely related to strains isolated from mayonnaise (Fig. 1) confirming the source of outbreak is due to consumption of contaminated mayonnaise.

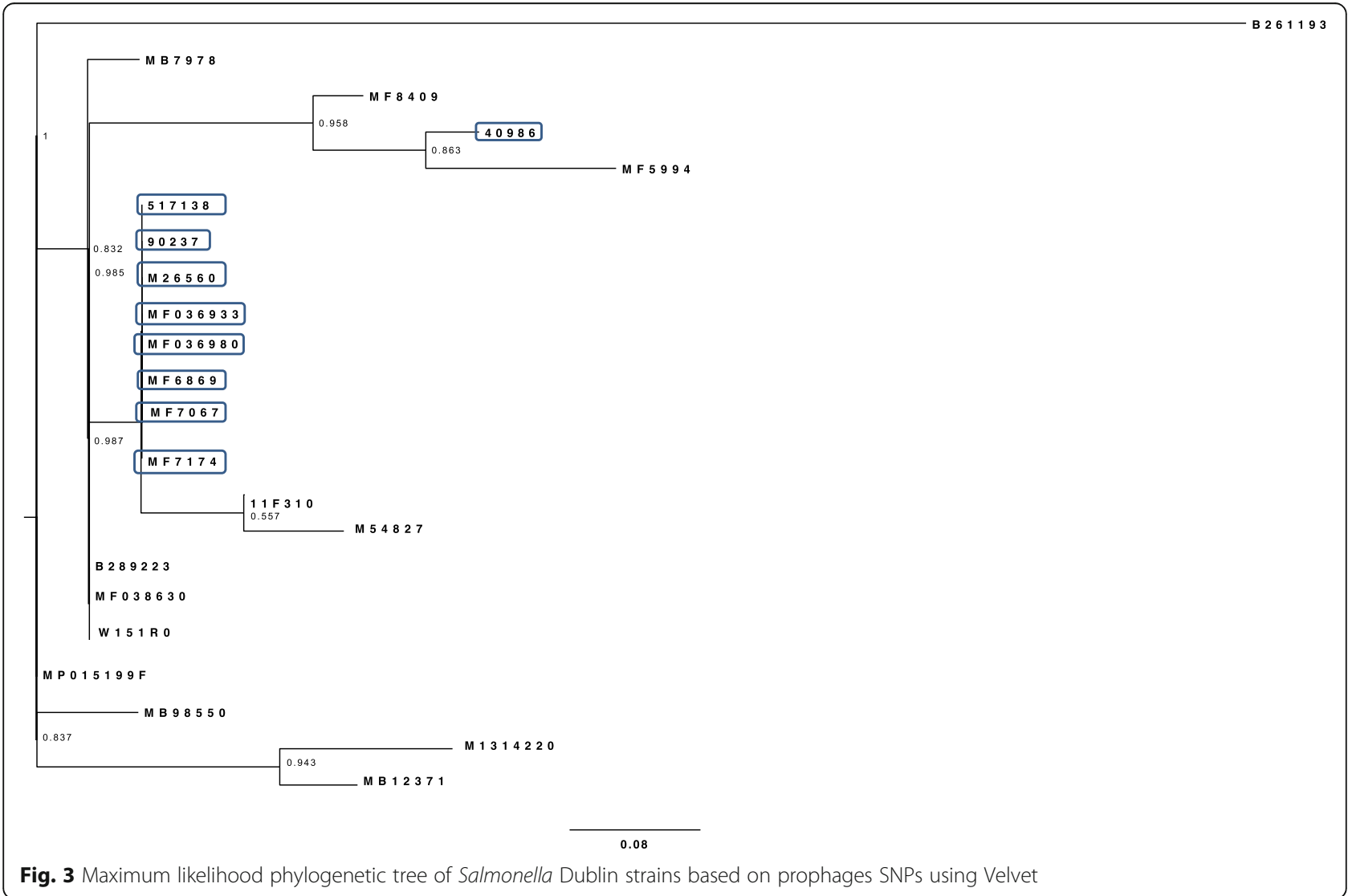


The outbreak isolates of Salmonella Dublin were closely related to each other (Fig. 2) and distinct from the non-outbreak isolates that were not readily distinguishable by PFGE. However, the source of Salmonella Dublin outbreak could not be determined and outbreak isolates showed high genetic divergence from the rawmilk cheese isolates related to other outbreaks occurred in France [10].

\section{MLST, rMLST, cgMLST and wgMLST}

As illustrated in Table 1, all Salmonella Dublin strains including the outbreak and non-outbreak strains showed identical MLST (type 10). Interestingly, outbreak isolates of Salmonella Dublin displayed identical rMLST (type 1429) however, some of the non-outbreak strains showed the same rMLST. Moreover, the wgMLST was different among the outbreak strains however, the cgMLST was unique among outbreak strains and can easily separate the outbreak strain from the nonoutbreak strains including the 2011 historical isolate (11F310).

On the other hand, MLST, rMLST, cgMLST and wgMLST could not discriminate between the outbreak and non-outbreak strains of Salmonella Typhimurium as illustrated in Table 2.

\section{CRISPR typing}

All Salmonella Dublin isolates including outbreak and non-outbreak strains harbour one CRISPR locus and we observed 3 to 5 unique spacers for CRISPR1 locus. Identical spacers were detected among the outbreak and non-outbreak strains as shown in Table 3.

Interestingly, the number of spacers in three isolates (517,138, MF7067 and W151R0) changed from (4 spacers) based on Velvet to (5 spacers) based on SPAdes.

All Salmonella Typhimurium isolates harbour 3 CRISPR loci. Identical spacers were detected among the outbreak and non-outbreak strains as shown in Table 4. There was no difference between the numbers of spacers using different assemblers.

\section{Prophage sequence profiling}

All Salmonella Dublin strains including the outbreak strains are lysogenic for three prophages (Gifsy_2, 118970_sal3 and RE_2010). However, phylogenetic analyses of Salmonella Dublin strains based on the SNPs of prophages showed that outbreak strains are intermixed with the non-outbreak strains based on velvet assembler (Fig. 3) and SPAdes assembler (Fig. 4).

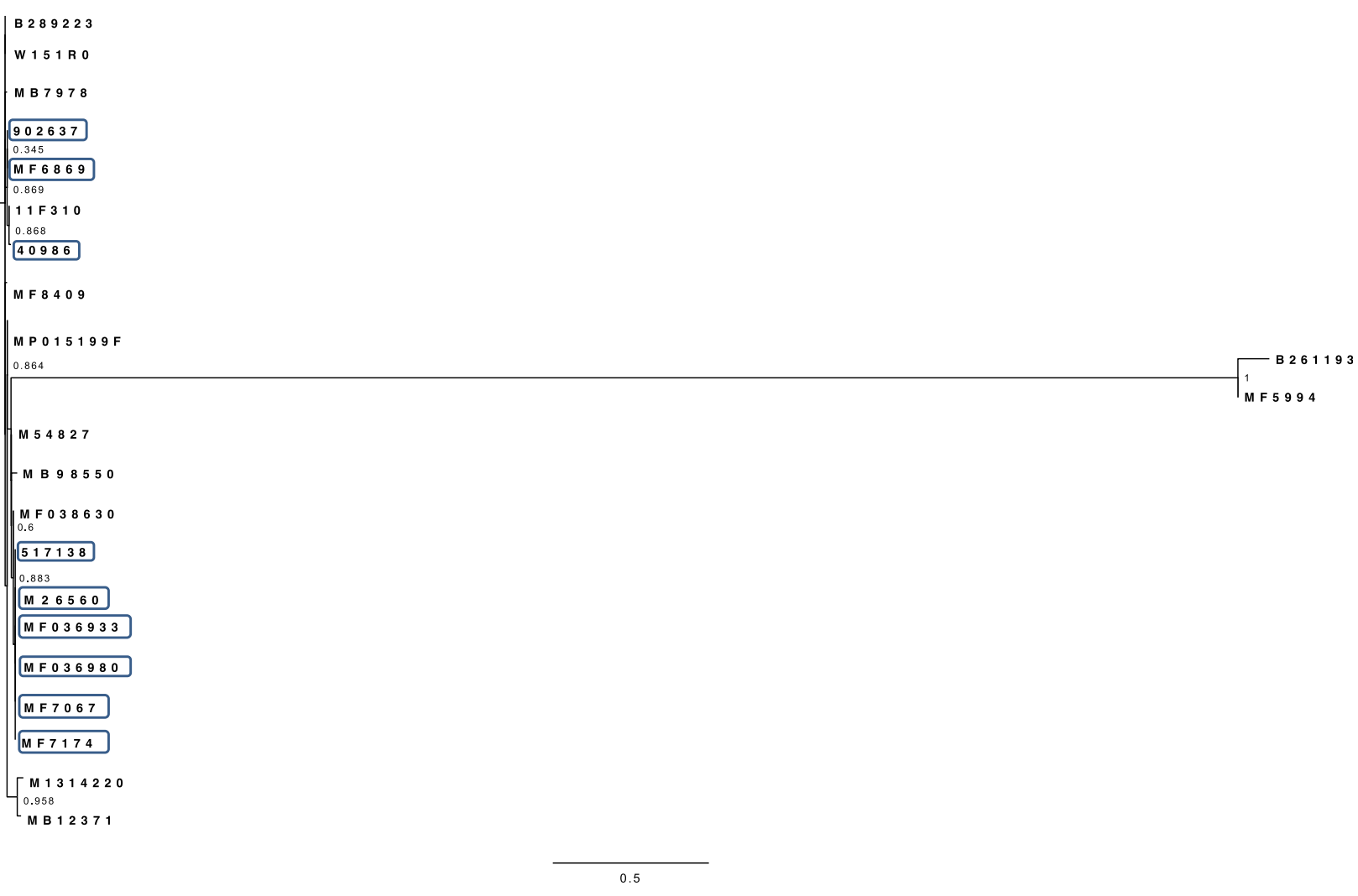

Fig. 4 Maximum likelihood phylogenetic tree of Salmonella Dublin strains based on prophages SNPs using SPAdes 
All Salmonella Typhimurium genomes assembled by SPAdes revealed the presence of four prophages in all outbreak and non-outbreak strains including the three Salmonella prophages (Gifsy 2, RE-2010, and 118970_ sal3) and the Edwardsiella specific phage (GF-2).

On the other hand, Salmonella Typhimurium genomes assembled by Velvet were lysogenic for two Salmonella specific prophages (Gifsy 2 and RE-2010). All strains except one outbreak isolate (H132940750) harbour Salmonella 118970_sal3 phage.

Interestingly, all strains harbour Edwardsiella GF-2 prophage except three outbreak isolates (H132940748, H133000645 and H133060376).

Phylogenetic analyses of Salmonella Typhimurium strains based on the SNPs of prophages showed that outbreak strains are intermixed with the non-outbreak strains using velvet assembler (Fig. 5) and using SPAdes assembler (Fig. 6).

\section{Plasmid typing}

All outbreak and non-outbreak strains of Salmonella Dublin harbour identical plasmid type (except three non-outbreak isolates; M1314220, MB12371 and B261193) as shown in Table 5.

Same plasmids were determined using Velvet and SPAdes assemblers.
All outbreak and non-outbreak isolates of Salmonella Typhimurium harbour 3 plasmids (pATCC14028, plasmid: 4 and pSE81-1705) except the outbreak strain H133300609 which did not carry plasmid pATCC14028 but it harbours a different plasmid (pSLT_VNP20009) instead (Table 6).

\section{Antibiotic resistance profile}

All Salmonella Dublin isolates including the outbreak and non-outbreak strains are resistant to aminoglycosides due to the acquisition of the $a a c\left(6^{\prime}\right)$-Iaa gene. No mutations were detected against $\operatorname{gyr} A$ and $\operatorname{parC}$ genes in all isolates except one isolate (MF038630) that carried a nonsynonyms mutation within the gyrase protein and it is associated with bacterial resistance to nalidixic acid (Table 7).

All the Salmonella Typhimurium isolates of both the outbreak and non-outbreak group are resistant to aminoglycosides due to the acquisition of the "aac(6')-Iaa gene". No known mutations were detected against gyrA and $\operatorname{parC}$ (Table 8).

\section{Discussion}

Salmonellosis is one of the most common foodborne diseases worldwide and has been associated with high morbidity and mortality rates. It is estimated that over

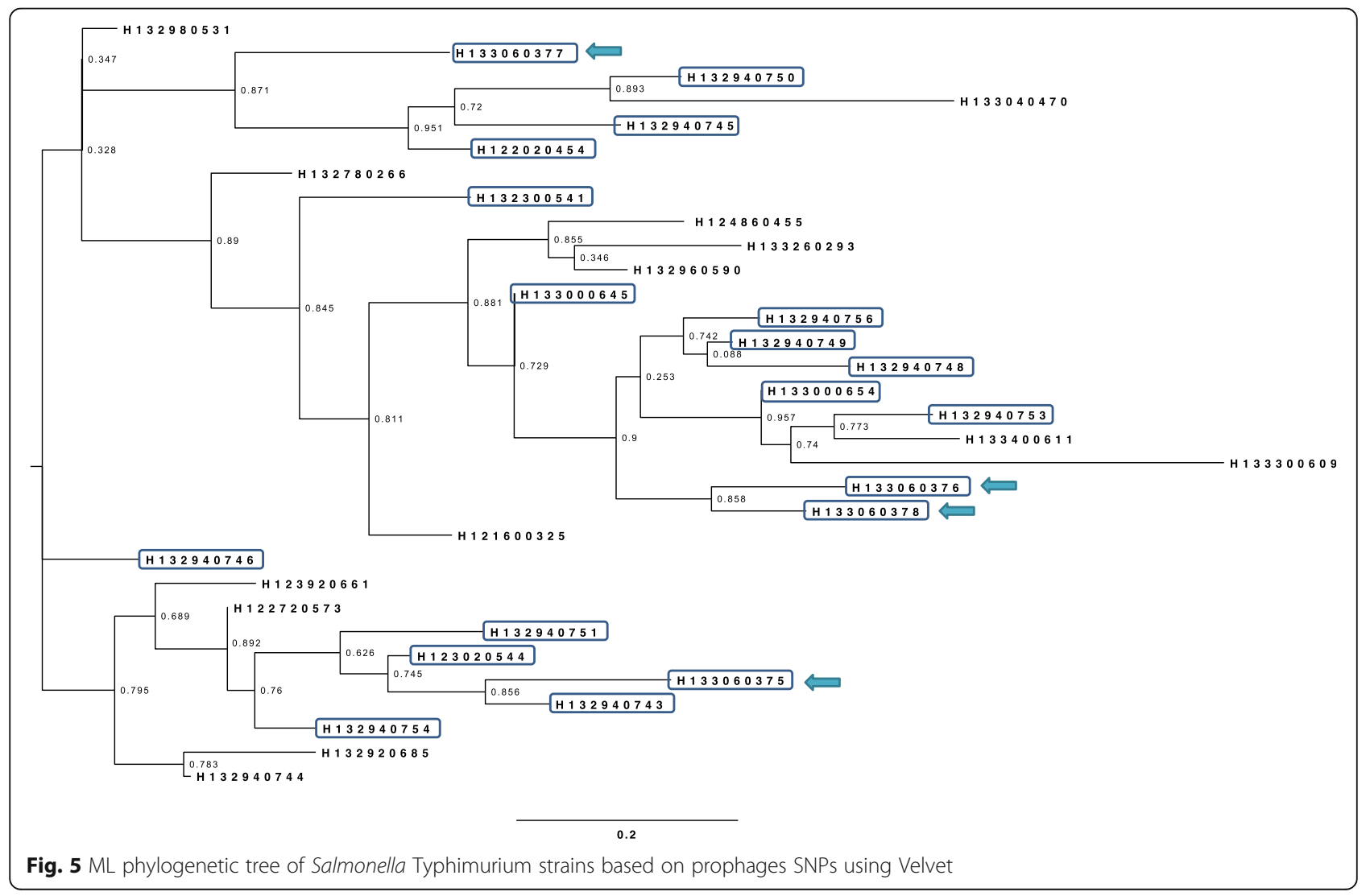




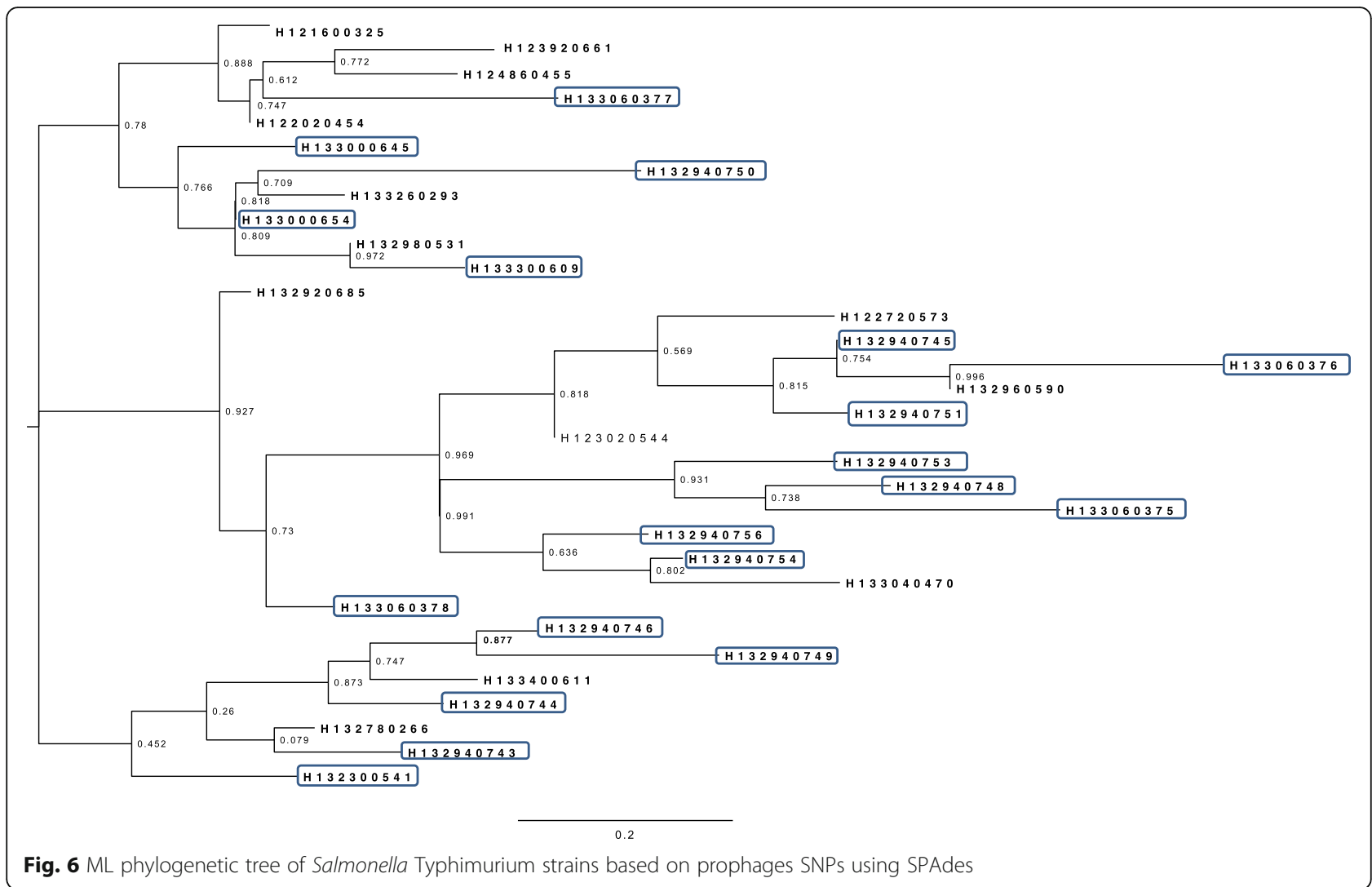

680,000 humans throughout the world are killed each year by iNTS. The most predominant iNTS serovars are Typhimurium, Enteritidis and Dublin [13, 14]. It is therefore very crucial to use accurate, reliable and highly discriminative subtyping methods for epidemiological surveillance and outbreak investigation.

Although PFGE is considered as current gold standard for all Salmonella serotypes, it has its limitations moreover, variation between laboratories has been reported when identifying the source of infection and discriminating between the outbreak and nonoutbreak isolates [15].

Other phenotypic tools such as phage typing and antimicrobial resistance profiling have been crucial in the outbreak investigations $[15,16]$. Furthermore, MLVA has been used to distinguish between genetically closely related strains and trace back the sources of disease outbreaks related to food $[15,17]$.

Genotypic approaches have ameliorated the methods for carrying out outbreak investigation and epidemiological surveillance [18]. The advent of whole genome sequencing (WGS) has opened the possibilities to enhance the typing approaches for outbreak investigation and epidemiological surveillance. In our study, WGS data have been analyzed to test the suitability of different approaches as subtyping tool for Salmonella enterica surveillance. We therefore carried out retrospective investigation of two different outbreaks of Salmonella Typhimurium and Salmonella Dublin that occurred in 2013 in UK and Ireland respectively $[6,19]$ using different WGS-subtyping methods.

In this study, single nucleotide polymorphism (SNP)-based cluster analysis of Salmonella Typhimurium genomes revealed well supported clades, that were concordant with epidemiologically defined outbreak and confirmed the source of outbreak is due to consumption of contaminated mayonnaise. Although SNP-analyses of Salmonella Dublin genomes confirmed the outbreak, however the source of infection could not be determined.

On the other the WGS-subtyping methods including MLST, rMLST, wgMLST, cgMLST showed limited discrimination for the outbreak and non-outbreak isolates of Salmonella Typhimurium strains. However, cgMLST defined the genetic relatedness among Salmonella Dublin isolates more precisely and confirmed there is no relation among the 2013 outbreak isolates and the 2011 historical isolate (11F310) of Salmonella Dublin.

It was reported that MLST might not be the most suitable epidemiological tool [20] but it is best for 
analyzing the genetic diversity of the strain and analyze the core and conserved genes of pathogens that are of public importance.

The cgMLST bridges the classic MLST with the novel WGS-based approach since it combines the discriminatory power of MLST with large-scale data obtained from WGS enabling to exploit a considerable number of gene targets throughout the bacterial genome which would maximize the quality and resolution for surveillance and research works.

A recent study showed that cgMLST has shown the robustness of cgMLST as a tool to investigate multi-country outbreak of Salmonella Enteritidis in Europe [21].

The difference between the cgMLST and wgMLST is that unlike cgMLST, wgMLST indexes the variation of pre-defined set of genes from both core and accessory genes [22]. Another retrospective study on 8 different outbreaks associated with verotoxigenic Escherichia coli (VTEC) O157:H7 in Canada showed that wgMLST provided higher discrimination than PFGE and MLVA [23].

Research studies have shown that cgMLST and wgMLST are viable typing methods for outbreak surveillance. In our study, cgMLST proved to provide higher discriminatory resolution for differentiating Salmonella Dublin isolates of outbreak group from the nonoutbreak group. However, both cgMLST and wgMLST were unsuccessful in differentiating outbreak-related Salmonella Typhimurium isolates from outbreakunrelated isolates.

Bacterial genome comprises a considerable amount (10 to $20 \%$ ) of prophages integrated in their core genome [24]. Prophages harbor genes for antimicrobial resistance, virulence and toxins which contribute to the genetic diversity of bacterial strains making

Table 5 Distribution of plasmids among outbreak and non-outbreak strains of Salmonella Dublin

\begin{tabular}{|c|c|c|c|c|c|c|}
\hline & $\begin{array}{l}\text { pSA19992307 } \\
\text { (NZ_CP030208) }\end{array}$ & $\begin{array}{l}\text { pSE81-1705 } \\
\text { (NZ_CP018654) }\end{array}$ & $\begin{array}{l}\text { Plasmid: } 4 \\
\text { (LN829404) }\end{array}$ & $\begin{array}{l}\text { pATCC39184 } \\
\text { (NZ_CP019180) }\end{array}$ & $\begin{array}{l}\text { pSDU2-USMARC-69807 } \\
\text { (NZ_CP032381) }\end{array}$ & $\begin{array}{l}\text { Plasmid: } 3 \\
\text { (NZ_LN868945) }\end{array}$ \\
\hline \multicolumn{7}{|l|}{ Outbreak strains: } \\
\hline 902,637 & Present & Present & Present & Absent & Absent & Absent \\
\hline MF036933 & Present & Present & Present & Absent & Absent & Absent \\
\hline MF036980 & Present & Present & Present & Absent & Absent & Absent \\
\hline 517,138 & Present & Present & Present & Absent & Absent & Absent \\
\hline MF6869 & Present & Present & Present & Absent & Absent & Absent \\
\hline M26560 & Present & Present & Present & Absent & Absent & Absent \\
\hline MF7067 & Present & Present & Present & Absent & Absent & Absent \\
\hline MF7174 & Present & Present & Present & Absent & Absent & Absent \\
\hline 40,986 & Present & Present & Present & Absent & Absent & Absent \\
\hline \multicolumn{7}{|c|}{ Non-outbreak strains: } \\
\hline MF038630 & Present & Present & Present & Absent & Absent & Absent \\
\hline M1314220 & Absent & Present & Present & Present & Absent & Absent \\
\hline M54827 & Present & Present & Present & Absent & Absent & Absent \\
\hline MB12371 & Absent & Present & Present & Present & Absent & Absent \\
\hline MF5994 & Present & Present & Present & Absent & Absent & Absent \\
\hline MB7978 & Present & Present & Present & Absent & Absent & Absent \\
\hline B289223 & Present & Present & Present & Absent & Absent & Absent \\
\hline $11 \mathrm{~F} 310$ & Present & Present & Present & Absent & Absent & Absent \\
\hline MB98550 & Present & Present & Present & Absent & Absent & Absent \\
\hline MF8409 & Present & Present & Present & Absent & Absent & Absent \\
\hline W151R0 & Present & Present & Present & Absent & Absent & Absent \\
\hline B261193 & Absent & Present & Present & Absent & Present & Absent \\
\hline MP015199F & Present & Present & Present & Absent & Absent & Absent \\
\hline Food strains: & & & & & & Absent \\
\hline${ }^{\mathrm{a}}$ 2014LSAL02972 & Present & Present & Absent & Absent & Absent & Present \\
\hline a2015LSAL00258 & Present & Present & Absent & Absent & Absent & Present \\
\hline
\end{tabular}

${ }^{\mathrm{a}}$ Salmonella Dublin strains isolated from raw milk cheeses related to other outbreaks occurred in France [10] 
prophages a potential marker for discriminating Salmonella serovars [25]. However, one of the limitations of using prophage sequence profiles for Salmonella subtyping is the sensitivity and accuracy of the assembly as some prophage regions might be lost during assembly. We used two different denovo assemblers (SPAdes and Velvet) and found that prophage sequence profiling could not differentiate between the outbreak and non-outbreak isolates.

Recent studies have suggested that high throughput CRISPR typing has the potential to be used for epidemiological surveillance and investigation of Salmonella outbreaks [26, 27]. However, in our study, we detected identical spacers among outbreak and non-

Table 6 Distribution of plasmids among outbreak and non-outbreak strains of Salmonella Typhimurium

\begin{tabular}{|c|c|c|c|c|}
\hline Strain ID & $\begin{array}{l}\text { pATCC14028 } \\
\text { (NZ_CP034231) }\end{array}$ & $\begin{array}{l}\text { Plasmid: } 4 \\
\text { (LN829404) }\end{array}$ & $\begin{array}{l}\text { PSE81-1705 } \\
\text { (NZ_CP018654) }\end{array}$ & $\begin{array}{l}\text { PSLT_VNP20009 } \\
\text { (NZ_CP008745) }\end{array}$ \\
\hline \multicolumn{5}{|l|}{ Food strains: } \\
\hline${ }^{\mathrm{a}} \mathrm{H} 133060375$ & Present & Present & Present & Absent \\
\hline${ }^{\mathrm{a}} \mathrm{H} 133060376$ & Present & Present & Present & Absent \\
\hline${ }^{\mathrm{a}} \mathrm{H} 133060377$ & Present & Present & Present & Absent \\
\hline${ }^{\mathrm{a}} \mathrm{H} 133060378$ & Present & Present & Present & Absent \\
\hline \multicolumn{5}{|l|}{ Outbreak strains: } \\
\hline H132300541 & Present & Present & Present & Absent \\
\hline H132940743 & Present & Present & Present & Absent \\
\hline H132940744 & Present & Present & Present & Absent \\
\hline H132940745 & Present & Present & Present & Absent \\
\hline H132940746 & Present & Present & Present & Absent \\
\hline H132940748 & Present & Present & Present & Absent \\
\hline H132940749 & Present & Present & Present & Absent \\
\hline H132940750 & Present & Present & Present & Absent \\
\hline H132940751 & Present & Present & Present & Absent \\
\hline H132940753 & Present & Present & Present & Absent \\
\hline H132940754 & Present & Present & Present & Absent \\
\hline H132940756 & Present & Present & Present & Absent \\
\hline H133000645 & Present & Present & Present & Absent \\
\hline H133000654 & Present & Present & Present & Absent \\
\hline H133300609 & Absent & Present & Present & Present \\
\hline \multicolumn{5}{|c|}{ Non-outbreak strains: } \\
\hline H121600325 & Present & Present & Present & Absent \\
\hline H122020454 & Present & Present & Present & Absent \\
\hline H122720573 & Present & Present & Present & Absent \\
\hline H123020544 & Present & Present & Present & Absent \\
\hline H123920661 & Present & Present & Present & Absent \\
\hline H124860455 & Present & Present & Present & Absent \\
\hline H132780266 & Present & Present & Present & Absent \\
\hline H132920685 & Present & Present & Present & Absent \\
\hline H132960590 & Present & Present & Present & Absent \\
\hline H132980531 & Present & Present & Present & Absent \\
\hline H133040470 & Present & Present & Present & Absent \\
\hline H133260293 & Present & Present & Present & Absent \\
\hline H133400611 & Present & Present & Present & Absent \\
\hline
\end{tabular}

${ }^{a}$ Strains of Salmonella Typhimurium isolated from mayonnaise 
Table 7 In silico analyses results of antimicrobial resistance genes and mutations within all Salmonella Dublin strains

\begin{tabular}{|c|c|c|c|}
\hline Strain ID: & Acquired antibiotic resistance genes: & Mutations in gyrA gene: & Mutations in parC gene: \\
\hline \multicolumn{4}{|l|}{ Outbreak strains: } \\
\hline 902,637 & Aminoglycoside $\left(\operatorname{aac}\left(6^{\prime}\right)\right.$-laa $)$ & Absent & Absent \\
\hline MF036933 & Aminoglycoside $\left(\operatorname{aac}\left(6^{\prime}\right)\right.$-laa $)$ & Absent & Absent \\
\hline MF036980 & Aminoglycoside $\left(a a c\left(\sigma^{\prime}\right)\right.$-laa) & Absent & Absent \\
\hline 517,138 & Aminoglycoside $\left(\operatorname{aac}\left(6^{\prime}\right)\right.$-laa) & Absent & Absent \\
\hline MF6869 & Aminoglycoside $\left(a a c\left(\sigma^{\prime}\right)\right.$-laa) & Absent & Absent \\
\hline M26560 & Aminoglycoside $\left(a a c\left(6^{\prime}\right)\right.$-laa) & Absent & Absent \\
\hline MF7067 & Aminoglycoside $\left(a a c\left(\sigma^{\prime}\right)\right.$-laa) & Absent & Absent \\
\hline MF7174 & Aminoglycoside $\left(a a c\left(6^{\prime}\right)-l a a\right)$ & Absent & Absent \\
\hline 40,986 & Aminoglycoside $\left(\operatorname{aac}\left(6^{\prime}\right)\right.$-laa) & Absent & Absent \\
\hline \multicolumn{4}{|c|}{ Non-outbreak strains: } \\
\hline MF038630 & Aminoglycoside $\left(\operatorname{aac}\left(6^{\prime}\right)\right.$-laa) & Absent & Present \\
\hline M1314220 & Aminoglycoside $\left(a a c\left(6^{\prime}\right)\right.$-laa) & Absent & Absent \\
\hline M54827 & Aminoglycoside $\left(a a c\left(6^{\prime}\right)\right.$-laa $)$ & Absent & Absent \\
\hline MB12371 & Aminoglycoside $\left(\operatorname{aac}\left(6^{\prime}\right)\right.$-laa $)$ & Absent & Absent \\
\hline MF5994 & Aminoglycoside $\left(\operatorname{aac}\left(6^{\prime}\right)\right.$-laa $)$ & Absent & Absent \\
\hline MB7978 & Aminoglycoside (aac(6')-laa) & Absent & Absent \\
\hline B289223 & Aminoglycoside $\left(\operatorname{aac}\left(6^{\prime}\right)\right.$-laa) & Absent & Absent \\
\hline $11 F 310$ & Aminoglycoside $\left(a a c\left(6^{\prime}\right)\right.$-laa $)$ & Absent & Absent \\
\hline MB98550 & Aminoglycoside $\left(\operatorname{aac}\left(6^{\prime}\right)\right.$-laa) & Absent & Absent \\
\hline MF8409 & Aminoglycoside $\left(a a c\left(6^{\prime}\right)-l a a\right)$ & Absent & Absent \\
\hline W151R0 & Aminoglycoside $\left(\operatorname{aac}\left(6^{\prime}\right)\right.$-laa) & Absent & Absent \\
\hline B261193 & Aminoglycoside $\left(a a c\left(6^{\prime}\right)\right.$-laa) & Absent & Absent \\
\hline MP015199F & Aminoglycoside $\left(\operatorname{aac}\left(6^{\prime}\right)-l a a\right)$ & Absent & Absent \\
\hline \multicolumn{4}{|l|}{ Food strains: } \\
\hline a2014LSAL02972 & Aminoglycoside $\left(\operatorname{aac}\left(6^{\prime}\right)\right.$-laa) & Absent & Absent \\
\hline${ }^{\mathrm{a}}$ 2015LSAL00258 & Aminoglycoside $\left(\operatorname{aac}\left(\sigma^{\prime}\right)\right.$-laa) & Absent & Absent \\
\hline
\end{tabular}

${ }^{a}$ Salmonella Dublin strains isolated from raw milk cheeses related to other outbreaks occurred in France [10]

outbreak associated strains indicating that CRISPR typing is not useful for the surveillance of Salmonella enetrica outbreaks as we showed in our previous studies $[28,29]$ however, it might be useful for the discrimination among different Salmonella serovars.

Plasmid profiles and antimicrobial- susceptibility profiling have been used as an epidemiological tool since many decades. However, it was reported that analysis of plasmid profiles provided higher discrimination in the outbreak investigations than analysis of antimicrobial-susceptibility pattern [30, 31]. In our study both plasmid typing and in silico analysis of antibiotic resistance were unable to discriminate between the outbreak isolates and non-outbreak isolates.

In this study, we compared several retrospective WGS-based subtyping methods and we showed that
SNP-based cluster analysis is superior to other subtying methods to define the source of outbreak in realtime.

In conclusion, foodborne salmonellosis is an important concern for public health therefore, it is crucial to use accurate, reliable and highly discriminative subtyping methods for epidemiological surveillance and outbreak investigation. The rapid development of next-generation sequencing (NGS) technology and bioinformatics tools have enabled WGS of any bacterial strain feasible. Various typing tools have been proposed by using WGS data but currently, the adoption of WGS-based methods have proved to be difficult due to lack of standardization. There are many layers on obtaining WGS data and there is need of standardization from the type of sequencers used to 
Table 8 In silico analyses results of antimicrobial resistance genes and mutations within all Salmonella Typhimurium strains

\begin{tabular}{|c|c|c|c|}
\hline Strain ID & Acquired antibiotic resistance genes: & Mutations in gyrA gene: & Mutations in parC gene: \\
\hline \multicolumn{4}{|l|}{ Food strains: } \\
\hline${ }^{\mathrm{a}} \mathrm{H} 133060375$ & Aminoglycoside (aac(6')-laa) & Absent & Absent \\
\hline${ }^{\mathrm{a}} \mathrm{H} 133060376$ & Aminoglycoside (aac( $\left.6^{\prime}\right)$-laa) & Absent & Absent \\
\hline${ }^{\mathrm{a}} \mathrm{H} 133060377$ & Aminoglycoside (aac(6')-laa) & Absent & Absent \\
\hline${ }^{\mathrm{a}} \mathrm{H} 133060378$ & Aminoglycoside (aac(6')-laa) & Absent & Absent \\
\hline \multicolumn{4}{|l|}{ Outbreak strains: } \\
\hline H132940743 & Aminoglycoside (aac(6')-laa) & Absent & Absent \\
\hline H132940744 & Aminoglycoside (aac(6')-laa) & Absent & Absent \\
\hline H132940745 & Aminoglycoside (aac(6')-laa) & Absent & Absent \\
\hline $\mathrm{H} 132940746$ & Aminoglycoside (aac(6')-laa) & Absent & Absent \\
\hline H132940748 & Aminoglycoside (aac(6')-laa) & Absent & Absent \\
\hline H132940749 & Aminoglycoside (aac(6')-laa) & Absent & Absent \\
\hline H132940750 & Aminoglycoside (aac(6')-laa) & Absent & Absent \\
\hline H132940751 & Aminoglycoside (aac(6')-laa) & Absent & Absent \\
\hline H132940753 & Aminoglycoside (aac( $\left.6^{\prime}\right)$-laa) & Absent & Absent \\
\hline H132940754 & Aminoglycoside (aac(6')-laa) & Absent & Absent \\
\hline H132940756 & Aminoglycoside (aac(6')-laa) & Absent & Absent \\
\hline H133000645 & Aminoglycoside (aac(6')-laa) & Absent & Absent \\
\hline H133000654 & Aminoglycoside (aac(6')-laa) & Absent & Absent \\
\hline H133300609 & Aminoglycoside (aac( $\left.6^{\prime}\right)$-laa) & Absent & Absent \\
\hline \multicolumn{4}{|c|}{ Non-outbreak strains: } \\
\hline H121600325 & Aminoglycoside (aac( $\left.6^{\prime}\right)$-laa) & Absent & Absent \\
\hline H122020454 & Aminoglycoside (aac(6')-laa) & Absent & Absent \\
\hline H122720573 & Aminoglycoside (aac(6')-laa) & Absent & Absent \\
\hline H123020544 & Aminoglycoside (aac(6')-laa) & Absent & Absent \\
\hline H123920661 & Aminoglycoside (aac(6')-laa) & Absent & Absent \\
\hline $\mathrm{H} 124860455$ & Aminoglycoside (aac(6')-laa) & Absent & Absent \\
\hline H132780266 & Aminoglycoside (aac(6')-laa) & Absent & Absent \\
\hline H132920685 & Aminoglycoside (aac(6')-laa) & Absent & Absent \\
\hline H132960590 & Aminoglycoside (aac(6')-laa) & Absent & Absent \\
\hline H132980531 & Aminoglycoside (aac(6')-laa) & Absent & Absent \\
\hline $\mathrm{H} 133040470$ & Aminoglycoside (aac( $\left.6^{\prime}\right)$-laa) & Absent & Absent \\
\hline H133260293 & Aminoglycoside (aac(6')-laa) & Absent & Absent \\
\hline H133400611 & Aminoglycoside (aac(6')-laa) & Absent & Absent \\
\hline
\end{tabular}

${ }^{a}$ Strains of Salmonella Typhimurium isolated from mayonnaise

the bioinformatics analysis. Therefore, the emerging genetic analysis techniques should be combined with conventional phenotypic and molecular methods for routine surveillance and outbreak investigation until the WGS-based methods can be fully exploited, improved and standardized.

\section{Supplementary information}

Supplementary information accompanies this paper at https://doi.org/10. 1186/s42522-020-00016-5.

Additional file 1: Supplementary Table 1. Details of Salmonella Dublin strains analysed in this study. Supplementary Table 2. Details of Salmonella Typhimurium strains analysed in this study 


\section{Abbreviations}

CGE: Centre for Genomic Epidemiology; cgMLST: Core genome multilocus sequence typing; CRISPRs: Clustered regularly interspaced short palindromic repeats; iNTS: Invasive NTS; ML: Maximum likelihood; MLST: Multilocus sequence typing; MLVA: Multiple loci VNTR analysis; NGS: Next generation sequencing; NTS: Non-typhoidal Salmonella; PE: Paired end; PFGE: Pulsed field gel electrophoresis; rMLST: Ribosomal MLST; SNP: Single nucleotide polymorphism; wgMLST: Whole genome MLST; WGS: Whole genome sequence

\section{Acknowledgements}

Authors would like to thank Falling Walls Lab London for the invitation to present this research work. Dr Manal Mohammed is funded by a Quinton Hogg start-up award, University of Westminster.

\section{Authors' contributions}

MM: designed the study, contributed to data analyses, wrote the initial draft of the manuscript. ST: contributed to data analyses and contributed to manuscript writing. All authors reviewed and edited the manuscript. The authors read and approved the final manuscript.

\section{Authors' information}

MM is a lecturer of Medical Microbiology, School of Life Sciences, University of Westminster. ST is a MSc Medical Microbiology student at School of Life Sciences, University of Westminster.

\section{Funding}

MM is funded by a Quinton Hogg start-up award, University of Westminster.

\section{Availability of data and materials}

Available in supplementary Tables 1 and 2

\section{Ethics approval and consent to participate}

Not Applicable.

\section{Consent for publication}

Authors agreed to publish.

\section{Competing interests}

Not Applicable.

Received: 4 February 2020 Accepted: 1 May 2020

Published online: 06 July 2020

\section{References}

1. Jajere SM. A review of Salmonella enterica with particular focus on the pathogenicity and virulence factors, host specificity and antimicrobial resistance including multidrug resistance. Vet World. 2019;12(4):504-21.

2. $\quad$ Barnett R. Typhoid fever. Lancet. 2016;388(10059):2467.

3. Stanaway JD, Parisi A, Sarkar K, Blacker BF, Reiner RC, Hay SI, et al. The global burden of non-typhoidal salmonella invasive disease: a systematic analysis for the global burden of disease study 2017. Lancet Infect Dis. 2019; 19(12):1312-24.

4. Mohammed M, Cormican M. Whole genome sequencing provides insights into the genetic determinants of invasiveness in Salmonella Dublin. Epidemiol Infect. 2016;144(11):2430-9.

5. Tennant SM, MacLennan CA, Simon R, Martin LB, Khan MI. Nontyphoida salmonella disease: current status of vaccine research and development. Vaccine. 2016;34(26):2907-10

6. Orzechowska B, Mohammed M. The war between bacteria and bacteriophages. Growing and handling of bacterial cultures Rijeka: IntechOpen; 2019. p.Ch. 6. http://www.intechopen.com/books/growingandhandling-of-bacterial-cultures/the-war-between-bacteria-\&-bacteriophages.

7. Crump JA, Mintz ED. Global trends in typhoid and paratyphoid fever. Clin Infect Dis. 2010:50(2):241-6.

8. Majowicz SE, Musto J, Scallan E, Angulo FJ, Kirk M, O'Brien SJ, et al. The global burden of nontyphoidal salmonella gastroenteritis. Clin Infect Dis. 2010;50(6):882-9.

9. Mohammed M, Delappe N, O'Connor J, McKeown P, Garvey P, Cormican M. Whole genome sequencing provides an unambiguous link between
Salmonella Dublin outbreak strain and a historical isolate. Epidemiol Infect. 2016:144(3):576-81.

10. Cadel-Six S, Vignaud ML, Mohammed M. Draft genome sequences of Salmonella enterica subsp. enterica Serovar Dublin strains from St. Nectaire and Morbier cheeses characterized by multilocus variable-number tandemrepeat analysis profiles associated with two fatal outbreaks in France. Microbiol Resour Announc. 2019;8(1). https://doi.org/10.1128/MRA.01361-18 eCollection 2019 Jan.

11. Ford L, Wang Q, Stafford R, Ressler KA, Norton S, Shadbolt C, et al. Seven Salmonella Typhimurium outbreaks in Australia linked by trace-Back and whole genome sequencing. Foodborne Pathog Dis. 2018;15(5):285-92.

12. Ashton PM, Peters T, Ameh L, McAleer R, Petrie S, Nair S, et al. Whole genome sequencing for the retrospective investigation of an outbreak of Salmonella Typhimurium DT 8. PLoS Curr. 2015;7. https://doi.org/10.1371/ currents.outbreaks.2c05a47d292f376afc5a6fcdd8a7a3b6.

13. Gordon MA. Invasive nontyphoidal salmonella disease: epidemiology, pathogenesis and diagnosis. Curr Opin Infect Dis. 2011;24(5):484-9.

14. Mohammed M, Vignaud ML, Cadel-Six S. Whole-genome sequences of two Salmonella enterica Serovar Dublin strains that harbor the viaA, viaB, and ompB loci of the vi antigen. Microbiol Resour Announc. 2019;8(14). https:// doi.org/10.1128/MRA.00028-19.

15. Lienemann T, Kyyhkynen A, Halkilahti J, Haukka K, Siitonen A. Characterization of Salmonella Typhimurium isolates from domestically acquired infections in Finland by phage typing, antimicrobial susceptibility testing, PFGE and MLVA. BMC Microbiol. 2015;15:131-8.

16. Mohammed $\mathrm{M}$, Cormican $\mathrm{M}$. Whole genome sequencing provides possible explanations for the difference in phage susceptibility among two Salmonella Typhimurium phage types (DT8 and DT30) associated with a single foodborne outbreak. BMC Res Notes. 2015;8(1):728.

17. Vignaud ML, Cherchame E, Marault M, Chaing E, Le Hello S, Michel V, et al. MLVA for Salmonella enterica subsp. enterica Serovar Dublin: development of a method suitable for inter-laboratory surveillance and application in the context of a raw Milk cheese outbreak in France in 2012. Front Microbiol. 2017;8:295.

18. Ferrari RG, Panzenhagen PHN, Conte-Junior CA. Phenotypic and genotypic eligible methods for Salmonella Typhimurium source tracking. Front Microbiol. 2017:8:2587.

19. Mohammed M, Le Hello S, Leekitcharoenphon P, Hendriksen R. The invasome of Salmonella Dublin as revealed by whole genome sequencing. BMC Infect Dis. 2017;17(1):544-X.

20. Sails AD, Swaminathan B, Fields PI. Utility of multilocus sequence typing as an epidemiological tool for investigation of outbreaks of gastroenteritis caused by Campylobacter jejuni. J Clin Microbiol. 2003;41(10):4733-9.

21. Pearce ME, Alikhan NF, Dallman TJ, Zhou Z, Grant K, Maiden MCJ. Comparative analysis of core genome MLST and SNP typing within a European Salmonella serovar Enteritidis outbreak. Int J Food Microbiol. 2018, 274:1-11.

22. Henri C, Leekitcharoenphon P, Carleton HA, Radomski N, Kaas RS, Mariet JF, et al. An assessment of different genomic approaches for inferring phylogeny of listeria monocytogenes. Front Microbiol. 2017;8:2351.

23. Rumore J, Tschetter L, Kearney A, Kandar R, McCormick R, Walker M, et al. Evaluation of whole-genome sequencing for outbreak detection of Verotoxigenic Escherichia coli O157:H7 from the Canadian perspective. BMC Genomics. 2018;19(1):870-3.

24. Manal M. Who fights whom? - understanding the complex dynamics of bacteria phage interaction using Anderson phage typing system. J Infect Dis Therapy. 1970;6(3):1-2.

25. Mottawea W, Duceppe MO, Dupras AA, Usongo V, Jeukens J, Freschi L, et al Salmonella enterica prophage sequence profiles reflect genome diversity and can be used for high discrimination subtyping. Front Microbiol. 2018;9: 836.

26. Fabre L, Zhang J, Guigon G, Le Hello S, Guibert V, Accou-Demartin M, et al. CRISPR typing and subtyping for improved laboratory surveillance of salmonella infections. PLoS One. 2012;7(5):e36995.

27. Shariat N, Dudley EG. CRISPRs: molecular signatures used for pathogen subtyping. Appl Environ Microbiol. 2014;80(2):430-9.

28. Mohammed M. Phage typing or CRISPR typing for epidemiological surveillance of Salmonella Typhimurium? BMC Res Notes. 2017;10(1):578

29. Mohammed M. Old school wins: outbreak investigation of foodborne salmonellosis; 2018. Available at: http://atlasofscience.org/old-school-winsoutbreak-investigation-of-foodborne-salmonellosis/. Accessed 15 Jan 2020. 
30. Holmberg SD, Wachsmuth IK, Hickman-Brenner FW, Cohen ML. Comparison of plasmid profile analysis, phage typing, and antimicrobial susceptibility testing in characterizing Salmonella Typhimurium isolates from outbreaks. J Clin Microbiol. 1984:19(2):100-4.

31. Brunner F, Margadant A, Peduzzi R, Piffaretti JC. The plasmid pattern as an epidemiologic tool for Salmonella Typhimurium epidemics: comparison with the lysotype. J Infect Dis. 1983;148(1):7-11.

\section{Publisher's Note}

Springer Nature remains neutral with regard to jurisdictional claims in published maps and institutional affiliations.

Ready to submit your research? Choose BMC and benefit from:

- fast, convenient online submission

- thorough peer review by experienced researchers in your field

- rapid publication on acceptance

- support for research data, including large and complex data types

- gold Open Access which fosters wider collaboration and increased citations

- maximum visibility for your research: over $100 \mathrm{M}$ website views per year

At $\mathrm{BMC}$, research is always in progress.

Learn more biomedcentral.com/submissions 